\title{
On the analytical investigation of hybrid transmission NOMA (H-NOMA)
}

\author{
Huseyin Haci ${ }^{1[0000-0002-0720-479 X]}$ and Joydev Ghosh ${ }^{2[0000-0001-6000-0123]}$ \\ 1 Department of Electrical and Electronic Engineering, Near East University,Mersin \\ 10, Turkey, \\ 2 School of Computer Science and Robotics, National Research Tomsk Polytechnic \\ University, Tomsk, Russia \\ E-mail:huseyin.haci@neu.edu.tr and joydev.ghosh.ece@gmail.com
}

\begin{abstract}
Non-orthogonal multiple access (NOMA) is shown to be the optimal channel access method and a strong candidate to be employed at the fifth generation $(5 \mathrm{G})$ and beyond networks. This paper studies direct transmission (DT) and cooperative transmission (CT) modes of operations in NOMA communications and proposes an investigation on evolving a cooperative transmission NOMA (C-NOMA) into a Hybrid transmission NOMA (H-NOMA) that can be used for design and deployment of relay based wireless networks, such as networks for Internet of Things (IoT) applications.
\end{abstract}

Keywords: Rician Fading Channel, NOMA, Direct Transmission Mode, Cooperative Transmission Mode, Shannon Capacity, Cumulative Distribution Function (CDF)

\section{Introduction}

Smart world applications such as Internet of Things (IoT) and Cloud based applications require high-capacity and reliable data transmissions between endpoints and gateways[1]. Channel access method is a crucial technology that determines the performance of communication systems. Non-orthogonal multiple access (NOMA) is shown to be the capacity achieving channel access method [1]. Thus, NOMA is envisioned as a strong candidate for the channel access method of the fifth generation (5G) and beyond networks [3]. Cooperative communications is the concept of user-equipment (UE) not acting only the receiver of the data transmissions but also as a helper (i.e. relay for multi-hop communications). Cooperative communications can significantly increase the communications performance through the diversity gain [4]. Further, NOMA can be employed together with cooperative communications without need for any hardware change. Employment of NOMA can increase the diversity gain further, since each UE can relay multiple signals simultaneously be superimposing them on a single symbol [5]. This makes cooperative NOMA communications a popular research topic. [4] proposed a novel transmission scheme for cooperative NOMA communications where a priori information available at the user with better channel 
condition is exploited to assist in other NOMA users' symbol detection. It has been shown that the outage probability and diversity order achieved are significantly improved compared to conventional cooperative communications. [3] studied optimal power allocation for cooperative NOMA for cellular multipleinput multiple-output (MIMO) downlink transmissions. user pairing shown to decrease the complexity. The performance investigation showed that by coordinating inter-beam and inter-frequency power allocation, the cell-edge users' performance can be significantly improved with a minor trade-off to cell-average users' performance. [2] proposed a cooperative relay system using NOMA and a sub-optimal power allocation scheme that can approach to the optimal scheme's performance when SNR is high. A significant increase in spectral efficiency is demonstrated compared to the cooperative relay systems that use orthogonal multiple access.

Despite interesting research, all of the aforementioned researches studied Rayleigh fading channels. Rayleigh channels are a special category of Rician fading channels and can only be practical when there is no line-of-sight signal. However, in today's networks there are many Femtocell and other types of Smallcell base-stations deployed where there is line-of-sight signal with high probability. Thus providing mathematical analysis that can be realized for channels with line-of-sight signals is important. Rician fading is a more generalized channel model compared to Rayleigh fading and can be used for channel models where there is a line-of-sight signal. Accordingly, the contributions of this paper are listed as follows:

- This paper studies and derives mathematical analysis for H-NOMA communications for Rician fading channels.

- Also, proposes a performance investigation model that is developed by using the conditional modes of operations for NOMA communications. Cumulative distribution function (CDF) analyses are shown to match very well with the computer simulations.

The rest of the paper is organized as follows. In Section 2, the method is described. The results are provided in Section 3 and relevant discussion is done in Section 4. Section 5 concludes the paper.

\section{System Model}

In Figure 1, we assume a two-user NOMA scenario with a source $(S)$ and two users for downlink transmission, where $S_{1}$ is used to denote near user and $S_{2}$ is used to denote far user. Therefore, $S_{1}$ and $S_{2}$ are considered as strong and the weak users respectively. The performance is evaluated over Rician fading channels with the channel co-efficients $h_{1}, h_{2}, h_{3}$ for the links $S$ to $S_{1}, S$ to $S_{2}$ and $S_{1}$ to $S_{2}$, respectively, where $h_{i} \sim C N\left(0, \sigma_{i}\right)$.

The transmission principle of the proposed Hybrid transmission NOMA (HNOMA) scheme follows two conditional modes of transmissions, such as direct transmission (DT) mode and cooperative transmission (CT) mode. At first, S 


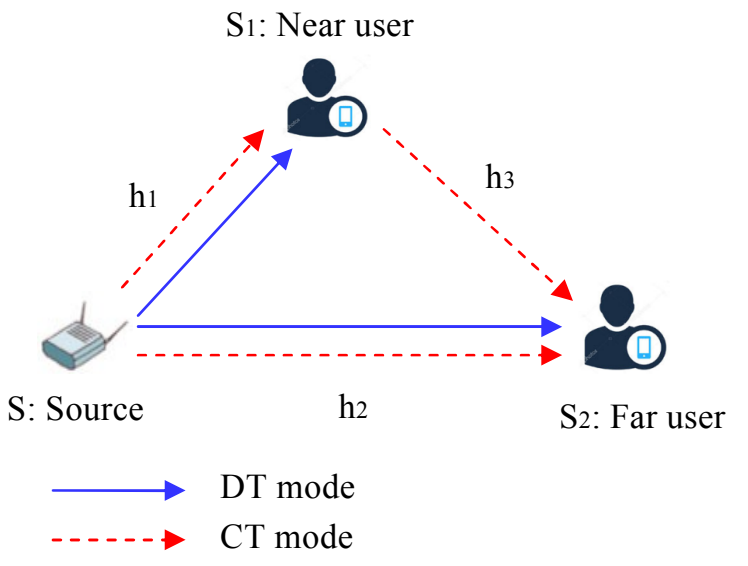

Fig. 1. The system model of H-NOMA transmission

broadcasts a reference signal to $S_{1}$ and $S_{2}$ to estimate the channel state information (CSI) of the received signals. By following the received signal, the comparison of estimated CSI (denoted by $\delta_{C S I}$ ) between $S_{2}$ and $S$ with set threshold ${ }^{3}$ has been computed and then $S_{2}$ decides whether it can successfully decodes its required symbol via direct transmission (DT) mode. If channel estimation is higher than $\gamma_{t h}$, then $S_{2}$ sends 1-bit binary acknowledgments ${ }^{4}$ to $S$ and $S_{1}$ to adapt DT mode of operation. And if channel estimation is lower than $\gamma_{t h}$ which implies it is unsuitable to decode its required symbol successfully, then $S_{2}$ sends 1-bit binary acknowledgments to $S$ and $S_{1}$ to adapt CT mode of operation.

\subsection{Channel Distribution}

In this paper, all estimated channel coefficients are modelled under Rician fading condition as it can mitigate self-interference (SI) link due to the short distance between antennas. Therefore, the probability density function (PDF) and cumulative distribution function (CDF) In the context of Rician fading, respectively, formulated as,

$$
f_{p d f}(\rho \mid v, \varphi)= \begin{cases}\frac{2 \rho}{\varphi} \exp \left(\frac{-\rho^{2}-v^{2}}{\varphi}\right) I_{0}\left(\frac{2 \rho v}{\varphi}\right) ; & \rho \geq 0, \varphi \geq 0 \\ \frac{1}{\sqrt{\pi \varphi}} \exp \left(-\frac{(\rho-v)^{2}}{\varphi}\right) ; & K \gg 1\end{cases}
$$

${ }^{3} \gamma_{t h}=2^{r_{0}}-1$, where $r_{0}$ denotes rate of information in bits $/ \mathrm{s} / \mathrm{Hz}$

4 1-bit binary acknowledgment,

$$
\beta_{A C K}= \begin{cases}1 ; & \delta_{C S I}>\gamma_{t h} \\ 0 ; & \delta_{C S I}<\gamma_{t h}\end{cases}
$$


where, $I_{0}($.$) is a zero-order modified Bessel function. The shape parameter, K=$ $\frac{v^{2}}{\varphi}$ defined as the ratio of the power contributions by line-of-sight path to the remaining multi-paths.

The Rician distribution can be written as, $R \sim \operatorname{Rice}(|v|, \varphi)$ if $R=\sqrt{X^{2}+Y^{2}}$ where $X \sim N(v \cos \theta, \varphi)$ and $Y \sim N(v \sin \theta, \varphi)$ are statistically independent normal random variables and $\theta=\left\{\frac{\pi}{2}, \frac{\pi}{3}, \frac{\pi}{4}\right\}$

\subsection{DT Mode of Operation:}

Initially $S$ broadcasts $\left(\sqrt{a_{1} P_{t}} x_{1}+\sqrt{a_{2} P_{t}} x_{2}\right)$ to $S_{1}$ and $S_{2}$, where $x_{1}$ and $x_{2}$ are the unit power signals for $S_{1}$ and $S_{2}$ respectively, $P_{t}$ denotes total transmit power, $a_{1}$ and $a_{2}$ are the corresponding power allocation coefficients of $S_{1}$ and $S_{2}$ respectively. We also assume that $a_{1} \geq a_{2}$ with $a_{1}+a_{2}=1$ to keep improved user fairness alongside quality of service (QoS). Hence, the received signals at $S_{1}$ and $S_{2}$ during DT mode of operation can be respectively given by,

$$
\begin{aligned}
& y_{1}=h_{1}\left(\sqrt{a_{1} P_{t}} x_{1}+\sqrt{a_{2} P_{t}} x_{2}\right)+N_{01}, \\
& y_{2}=h_{2}\left(\sqrt{a_{1} P_{t}} x_{1}+\sqrt{a_{2} P_{t}} x_{2}\right)+N_{02},
\end{aligned}
$$

where $N_{01}$ and $N_{02}$ denote the additive white Gaussian noise (AWGN) with zero mean and variance $\sigma^{2}$ at $S_{1}$ and $S_{2}$ respectively. The $S_{2}$ only decodes $x_{1}$ from (3) by treating $x_{2}$ as an unwanted signal, while $S_{1}$ acquires $x_{2}$ from (2) applying successive interference cancellation (SIC) technique. Therefore, the SINR for $x_{1}$ and SNR for $x_{2}$ at $S_{1}$ can be respectively expressed as,

$$
\begin{gathered}
\gamma_{1}=\frac{\left|h_{1}\right|^{2} a_{1} P_{t}}{\left|h_{1}\right|^{2} a_{2} P_{t}+\sigma^{2}} \\
\gamma_{2}=\frac{\left|h_{1}\right|^{2} a_{2} P_{t}}{\sigma^{2}}
\end{gathered}
$$

The SINR for $x_{1}$ at $S_{2}$ can be expressed as,

$$
\gamma_{3}=\frac{\left|h_{2}\right|^{2} a_{1} P_{t}}{\left|h_{2}\right|^{2} a_{2} P_{t}+\sigma^{2}}
$$

\subsection{CT Mode of Operation:}

In this mode of operation transmission is generally performed in two phases. From figure 1, the overlapping arrows between DT mode and CT mode are assumed to be $1^{\text {st }}$ phase, else $2^{\text {nd }}$ phase. In the $1^{\text {st }}$ phase, the SINR for $x_{1}$ and SNR for $x_{2}$ at $S_{1}$, and SINR for $x_{1}$ at $S_{2}$ are all that remain same as (4), (5) and (6). In the $2^{\text {nd }}$ phase, $S_{1}$ forwards the re-encoded $x_{2}$ to $S_{2}$. The received signal at $S_{2}$ in the $2^{\text {nd }}$ phase is given by,

$$
y_{3}=h_{3} \sqrt{P_{t}} x_{2}+N_{03}
$$


Hence, the SNR for $x_{2}$ at $S_{2}$ can be expressed as,

$$
\gamma_{4}=\frac{\left|h_{3}\right|^{2} P_{t}}{\sigma^{2}}
$$

\subsection{Shannon Capacity Analysis:}

Based on DT mode of operation we can determine the capacities $C_{1}^{D T}$ and $C_{2}^{D T}$ of $x_{1}$ and $x_{2}$ respectively as,

$$
\begin{gathered}
C_{1}^{D T}=\frac{B}{2} \min \left\{\log _{2}\left(1+\gamma_{1}\right), \log _{2}\left(1+\gamma_{3}\right)\right\} \\
C_{2}^{D T}=\frac{B}{2} \log _{2}\left(1+\gamma_{2}\right)
\end{gathered}
$$

Likewise, based on CT mode of operation we can determine the capacities $C_{1}^{C T}$ and $C_{2}^{C T}$ of $x_{1}$ and $x_{2}$ respectively as,

$$
\begin{aligned}
C_{1}^{C T} & =\frac{B}{2} \min \left\{\log _{2}\left(1+\gamma_{1}\right), \log _{2}\left(1+\gamma_{3}\right)\right\} \\
C_{2}^{C T} & =\frac{B}{2} \min \left\{\log _{2}\left(1+\gamma_{2}\right), \log _{2}\left(1+\gamma_{4}\right)\right\}
\end{aligned}
$$

By following the H-NOMA scheme, the total capacity of the systems can be expressed by,

$$
C_{\text {total }}=\frac{B}{2}\left[\beta_{A C K}\left\{C_{1}^{D T}+C_{2}^{D T}\right\}+\beta_{A C K}\left\{C_{1}^{C T}+C_{2}^{C T}\right\}\right]
$$

\subsection{CDF Analysis:}

With the consideration of $h_{i} \sim C N\left(0, \sigma_{1}\right)$, the probability of successfully decoding $x_{1}$ at $S_{1}$ can be expressed as,

$$
P\left(\gamma_{1}>\gamma_{t h}\right)=P\left(\left|h_{1}\right|^{2}>\frac{\gamma_{t h}}{a_{1} P_{t}-a_{2} P_{t}} \gamma_{t h}\right)= \begin{cases}e^{-\frac{\gamma_{t h}}{\left(a_{1}-a_{2} \gamma_{t h}\right) P_{t} \sigma_{1}^{2}}}, \gamma_{t h}<\frac{a_{1}}{a_{2}} \\ 0 \quad, \gamma_{t h}>\frac{a_{1}}{a_{2}}\end{cases}
$$

Now, the CDF of $\gamma_{1}$ is given by,

$$
f_{\gamma_{1}}=\frac{a_{1}}{\left(a_{1}-a_{2} \gamma_{t h}\right)^{2} P_{t} \sigma_{1}^{2}} e^{-\frac{\gamma_{t h}}{\left(a_{1}-a_{2} \gamma_{t h}\right) P_{t} \sigma_{1}^{2}}}
$$



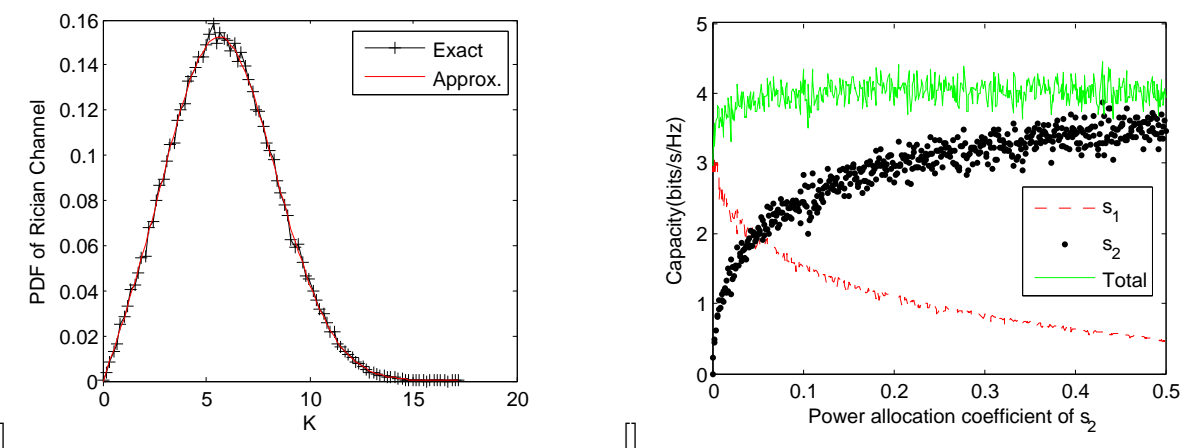

Fig. 2. PDF of Rician channel versus $K, 0 \leq K \leq 18$, for the exact and approximation expressions (Fig. 2a), The capacity (bits/s/Hz) versus power allocation coefficient of $s_{2}$ for the total system (Fig. 2b).
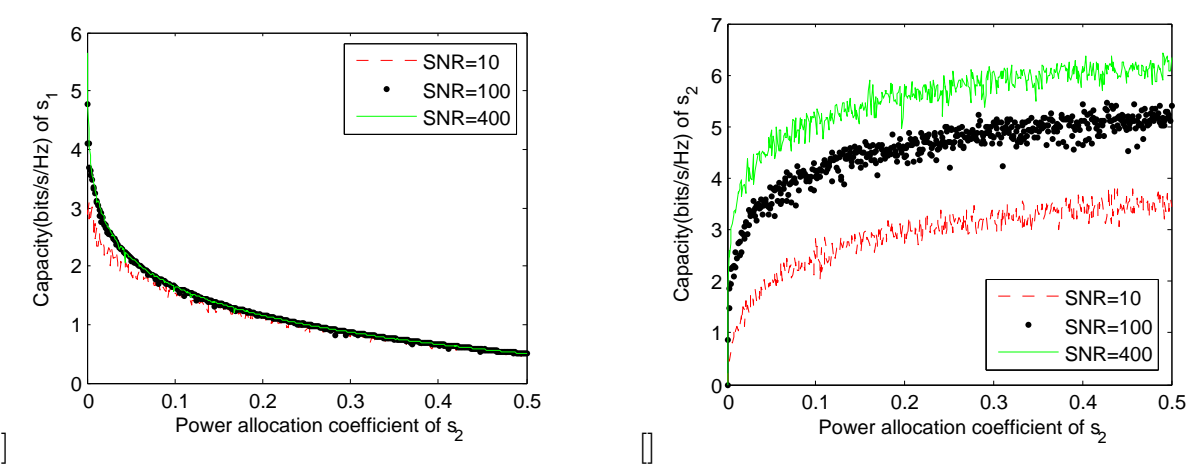

Fig. 3. The capacity of $s_{1}$ (bits/s/Hz) versus power allocation coefficient of $s_{2}$ changing from 0 to 0.5 for $\mathrm{SNR}=\{10,100,400\} \mathrm{dB}$ (Fig. 3a), The capacity (bits/s/Hz) versus power allocation coefficient ranging from 0 to 0.5 and received $\mathrm{SNR}=\{10,100,400\}$ $\mathrm{dB}$ of $s_{2}$ (Fig. $3 \mathrm{~b}$ ).

\section{Results and discussion}

Figure 2(a) shows the probability density function (PDF) of Rician channel versus $K$ for the exact expression and the approximation given by (1). $K$ is the ratio of the power contributions by line-of-sight signal to the remaining multipath signals. It can be seen from the figure that the both curves match very well for the whole considered region of $0 \leq K \leq 18$. This proves that the approximation can be used without significant divergence from the results that would be obtained by the use of exact expression.

Figure 2(b) shows the spectral efficiency, i.e. capacity in bits per seconds per Hertz, versus power allocation coefficient of $s_{2}$, changing from 0 to 0.5 , for the total system, $s_{1}$ and $s_{2}$. The total system capacity is seen to be stable with respect to the change in power allocation coefficient of $s_{2}$. This is because, as the power allocation coefficient increases the capacity of $s_{2}$ increases with a 

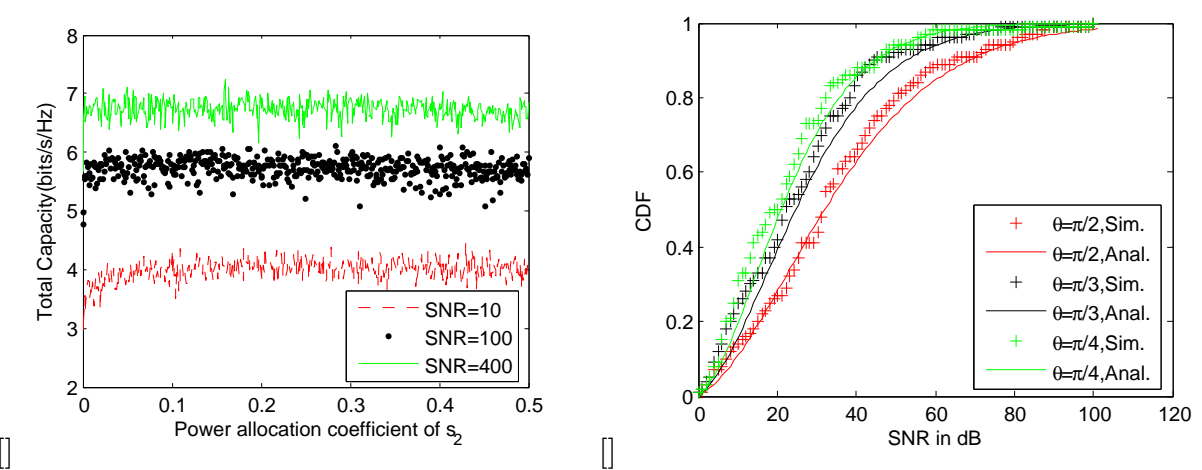

Fig. 4. Total capacity of the system (bits/s/Hz) versus power allocation coefficient ranging from 0 to 0.5 and received $\mathrm{SNR}=\{10,100,400\} \mathrm{dB}$ of $s_{2}$ (Fig. 4a), Computer simulation and analytical results for CDF of SNR for $=\left\{\frac{\pi}{2}, \frac{\pi}{3}, \frac{\pi}{4}\right\}$ (Fig. 4b).

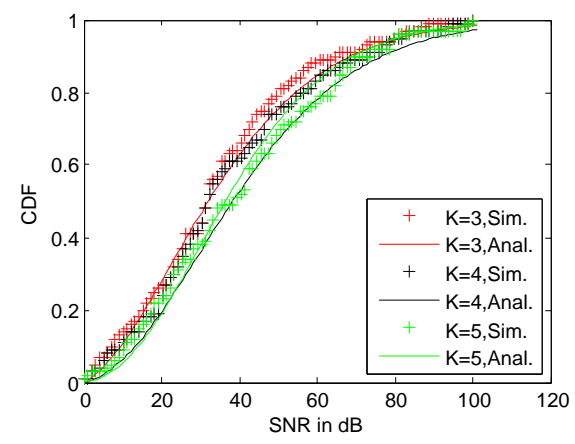

Fig. 5. Computer simulation and analytical results for CDF of SNR for $K=\{3,4,5\}$.

logarithmic scale and the capacity of $s_{1}$ decreases in a similar scale. It is clear that power allocation coefficient can be used to adjust the capacity of $s_{1}$ and $s_{2}$ without degrading the total system capacity.

Figure 3(a) displays the capacity of $s_{1}$ in bits/s/Hz versus power allocation coefficient of $s_{2}$ changing from 0 to 0.5 and received SNR of $s_{2}=\{10,100,400\}$ $\mathrm{dB}$. It can be seen that the capacity curve of $s_{1}$ is not much affected with respect to the change in SNR of $s_{2}$ from 10 to 100 and $400 \mathrm{~dB}$. This is because, by employing H-NOMA the diversity is increased at the receiver and the signal of $s_{2}$ can be successfully detected with high probability even with $S N R=10 \mathrm{~dB}$. And the interference from signal of $s_{2}$ to the signal of $s_{1}$ can be suppressed through successive interference cancellation (SIC) [1].

The capacity of $s_{2}(\mathrm{bits} / \mathrm{s} / \mathrm{Hz})$ versus its power allocation coefficient ranging from 0 to 0.5 and SNR of $=\{10,100,400\}$ dBis shown in figure $3(\mathrm{~b})$. As the SNR increases the capacity of $s_{2}$ increases with a higher degree of change from 10 to $100 \mathrm{~dB}$ compared to 100 to $400 \mathrm{~dB}$.This shows that the capacity of $s_{2}$ may 
saturate after a level of increase in its SNR, since the residual interference from $s_{1}$ may have an effect on this performance.

Figure 4(a) shows the total capacity of the system (bits/s/Hz) versus power allocation coefficient ranging from 0 to 0.5 and received $\mathrm{SNR}=\{10,100,400\}$ $\mathrm{dB}$ of $s_{2}$. In compliance with figure $2(\mathrm{~b})$, the total capacity of the system is shown to be independent of the power allocation coefficient since the capacity loss of $s_{1}$ is compensated with the capacity gain of $s_{2}$ as this coefficient increases. With the increase of the received SNR of $s_{2}$, the total capacity of the system increases due to $s_{2}$ achieving higher capacity and not interfering with the signal of $s_{1}$ through performing SIC.

Figure 4(b) shows the computer simulation and analytical results for CDF of SNR for $\theta=\left\{\frac{\pi}{2}, \frac{\pi}{3}, \frac{\pi}{4}\right\}$. It can be seen from the figure that both curves match very well for all the considered cases of $\theta$. Accordingly, mathematical analyses developed for Rician fading channel model are valid for wide range of channel scenarios. Further, it is clear that $\theta$ has a significant effect on the SNR performance.

Figure 5 shows the computer simulation and analytical results for the CDF of SNR for $K=\{3,4,5\}$. It is clear from the figure that for all the considered cases of $K$ the computer simulations and analytical results match well. Thus, the mathematical analyses developed are valid for a wide range of shape parameter (i.e. $K$ ) for Rician fading channel model. The change of $K$ has less effect on the CDF of SNR compared to the effect of $\theta$ shown by figure 4(b).

\section{Conclusion}

A set of operations and functions performed in the closed-form expressions that derive the Shannon capacity of the systems and CDF of SNR for the proposed HNOMA scheme. It is shown that the computer simulations and analytical results match very well. CDF analysis can be used as a tool for design and deployment of $5 \mathrm{G}$ and beyond networks where cooperative communications are useful, such as IoT applications.

\section{References}

1. Haci, H., Zhu, H., Wang, J.: Performance of Non-orthogonal Multiple Access With a Novel Asynchronous Interference Cancellation Technique. IEEE Trans. on Comm..65, 1319-1335 (2017).

2. Kim, J., Lee, I.: Capacity Analysis of Cooperative Relaying Systems Using NonOrthogonal Multiple Access.IEEE Communications Letters.19, 1949-1952(2015).

3. Hayashi, Y.,Kishiyama, Y., Higuchi,K.: Investigations on Power Allocation among Beams in Non-Orthogonal Access with Random Beamforming and Intra-Beam SIC for Cellular MIMO Downlink. In: 78th Vehicular Technology Conference (VTC Fall), pp. 1-5. IEEE, Las Ve-gas(2013).

4. Ding, Z., Peng, M., Poor,H. V.: Cooperative Non-Orthogonal Multiple Access in 5G Systems. IEEE Communications Letters.19, 1462-1465(2015). 